\title{
Safety and efficacy of transcranial direct current stimulation in upper extremity rehabilitation after tetraplegia: protocol of a multicenter randomized, clinical trial
}

\author{
Tarun Arora ${ }^{1}$, Kyle O'Laughlin' ${ }^{1}$, Kelsey Potter-Baker ${ }^{2}$, Steven Kirshblum ${ }^{3,4,5}$, Kevin Kilgore ${ }^{6,7,8}$, Gail F. Forrest ${ }^{3,5}$, Anne M. Bryden ${ }^{6,9}$, \\ Xiaofeng Wang ${ }^{10}$, M. Kristi Henzel iD $^{8,11}$, Manshi Li ${ }^{10}$, Kaitlin Perlic ${ }^{12}$, Mary Ann Richmond ${ }^{8,13}$, Svetlana Pundik ${ }^{8,14}$, Francois Bethoux ${ }^{15}$, \\ Frederick Frost ${ }^{15}$ and Ela B. Plow ${ }^{1,15} \bowtie$
}

(c) The Author(s), under exclusive licence to International Spinal Cord Society 2022

STUDY DESIGN: A multisite, randomized, controlled, double-blinded phase I/II clinical trial.

OBJECTIVE: The purpose of this clinical trial is to evaluate the safety, feasibility and efficacy of pairing noninvasive transcranial direct current stimulation (tDCS) with rehabilitation to promote paretic upper extremity recovery and functional independence in persons living with chronic cervical spinal cord injury (SCI).

SETTING: Four-site trial conducted across Cleveland Clinic, Louis Stokes Veterans Affairs Medical Center of Cleveland and MetroHealth Rehabilitation Rehabilitation Institute of Ohio, and Kessler Foundation of New Jersey.

METHODS: Forty-four adults (age $\geq 18$ years) with tetraplegia following cervical SCI that occurred $\geq 1$-year ago will participate. Participants will be randomly assigned to receive anodal tDCS or sham tDCS given in combination with upper extremity rehabilitation for 15 sessions each over 3-5 weeks. Assessments will be made twice at baseline separated by at least a 3-week interval, once at end-of-intervention, and once at 3-month follow-up.

PRIMARY OUTCOME MEASURE(S): Primary outcome measure is upper extremity motor impairment assessed using the Graded Redefined Assessment of Strength, Sensibility and Prehension (GRASSP) scale. Functional abilities will be assessed using Capabilities of Upper Extremity-Test (CUE-T), while functional independence and participation restrictions will be evaluated using the self-care domain of Spinal Cord Independent Measure (SCIM), and Canadian Occupational Performance Measure (COPM).

SECONDARY OUTCOME MEASURES: Treatment-associated change in corticospinal excitability and output will also be studied using transcranial magnetic stimulation (TMS) and safety (reports of adverse events) and feasibility (attrition, adherence etc.) will also be evaluated.

TRIAL REGISTRATION: ClincalTrials.gov identifier NCT03892746. This clinical trial is being performed at four sites within the United States: Cleveland Clinic (lead site), Louis Stokes Cleveland Veterans Affairs Medical Center (VAMC) and MetroHealth Rehabilitation Institute in Ohio, and Kessler Foundation in New Jersey. The U.S. Army Medical Research Acquisition Activity, 820 Chandler Street, Fort Detrick MD 21702-5014 is the awarding and administering acquisition office.

Spinal Cord (2022) 60:774-778; https://doi.org/10.1038/s41393-022-00768-z

\section{INTRODUCTION}

There are over 250,000 individuals living with traumatic spinal cord injury $(\mathrm{SCl})$ in the United States [1]. Tetraplegia is the most frequent $(67 \%)$ neurological outcome after a traumatic SCI [1]. Individuals with tetraplegia prioritize return of upper extremity (UE) function over any other lost function [2], yet the effects of UE rehabilitation are often slow and incomplete [3]. Weak and depressed physiologic excitability of residual corticomotor pathways likely contributes to incomplete recovery [4].

Enhancing the excitability of residual corticomotor pathways using brain stimulation holds promise for promoting functional recovery in $\mathrm{SCl}$ [5]. Transcranial direct current stimulation (tDCS) is a common noninvasive brain stimulation technique that involves application of weak direct electrical currents to modify cortical

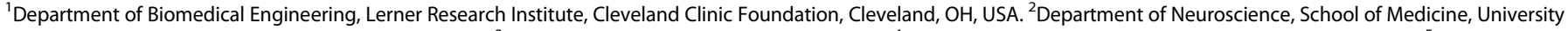

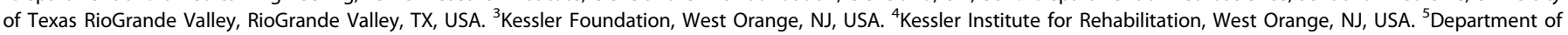

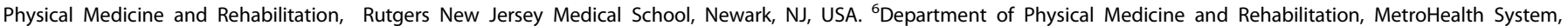

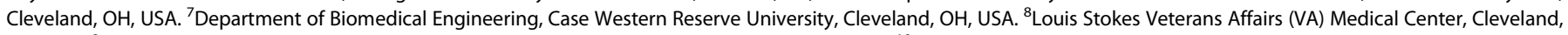

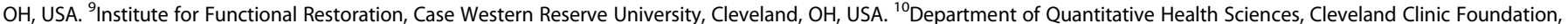

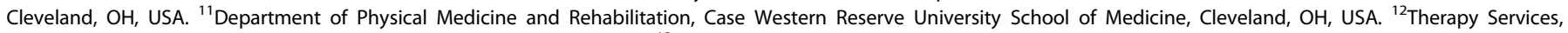

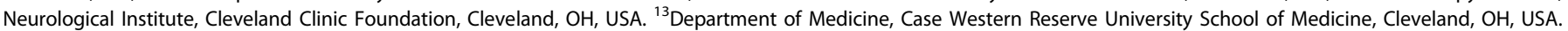

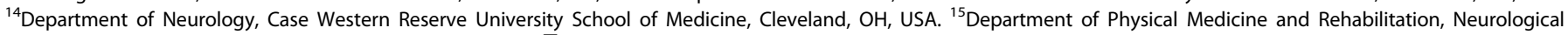
Institute, Cleveland Clinic Foundation, Cleveland, OH, USA. ${ }^{凶}$ email: plowe2@ccf.org 
Table 1. Inclusion and exclusion criteria.

\author{
Inclusion criteria \\ 1. Adults $\geq 18$ y old \\ 2. Traumatic cervical SCI \\ 3. Time since injury $\geq 1 \mathrm{y}$ \\ 4. Imbalance of muscle strength (at least 1 MRC difference) between biceps \\ and triceps such that \\ a. Weaker muscle $\mathrm{MRC}=1-3$ \\ b. Stronger muscle MRC $=3-5$
}

\section{Exclusion criteria}

1. Contraindications to TMS or tDCS

a. Pacemaker

b. Metal in skull

c. History of seizures

d. Pregnancy

2. Active ulcers

3. Traumatic brain injury, Rancho scale $<5$ or positive MRI/CT findings

4. Excessive tone/spasticity, Modified Ashworth Scale $>3$

5. Severe contractures or tissue shortening limited movement at elbow or wrist

6. Current participation in ongoing upper extremity therapies excitability [6]. Application of anodal tDCS can raise corticomotor excitability while application of cathodal tDCS can suppress corticomotor excitability [7]. Recent studies have tested the effects of pairing tDCS with rehabilitation in SCl with the goal of potentiating residual corticomotor excitability and enhancing rehabilitation outcomes [8-11]. In a pilot study, our group revealed that anodal tDCS combined with rehabilitation (tDCS + rehab) for ten sessions produces twice as much gain in muscle power as sham tDCS combined with rehabilitation (shamtDCS + rehab) and associated improvement in dexterity [8]. Effects of $t D C S+$ rehab are associated with gain in corticomotor excitability for weak muscle below the level of injury, assessed using transcranial magnetic stimulation (TMS). Other studies have demonstrated similar promise of combining anodal tDCS with UE motor training for persons with chronic tetraplegia [9-12]. Despite positive evidence, a recent meta-analysis of six studies concluded the effect of tDCS on motor function in $\mathrm{SCl}$ was marginal, associated with small effect sizes. A need for highquality randomized controlled trials (RCTs) evaluating long-term effects in larger samples was emphasized [13].

Our group is performing a multisite, phase $\mathrm{I} / \mathrm{Il}$ double-blinded RCT $(N=44)$ to evaluate the effectiveness (Aim 1$)$, neurophysiologic mechanisms (Aim 2) and safety and feasibility (Aim 3) of pairing tDCS with rehabilitation for recovery of UE function and independence in individuals with chronic tetraplegia. We hypothesize that $t D C S+r e h a b$ will promote greater improvements in strength, dexterity, functional abilities and independence, and larger reduction in participation restrictions compared to shamtDCS + rehab. We anticipate the effects of tDCS will be associated with gains in corticomotor excitability and output and evidence of cortical reorganization, assessed using TMS. We expect tDCS will be safe and feasible to use in combination with rehabilitation in individuals with chronic tetraplegia.

\section{METHODS}

\section{Participants and sample size}

We will enroll 44 adults ( $\geq 18$ years of age) with tetraplegia resulting from a traumatic cervical SCl that occurred $\geq 1$-year ago. Participants must have an imbalance of muscle strength between biceps and triceps on the moreaffected side, with the stronger muscle having Medical Research Council (MRC) grade of 3-5 and the weaker muscle having MRC grade at least one level lower (1-3) (Table 1). Muscle imbalance is characteristic of SCl and can provide important insight into corticomotor neurophysiology for relatively spared versus weaker muscles. Triceps due to its distal innervation is likely to be weaker than biceps in a typical C5-C6 injury. Study of the biceps-triceps muscle pair therefore is functionally relevant since differences in their strength can impair elbow-forearm motor control and interfere with functional activities and self-care tasks that involve transfers, pressure-relief and weight-shift maneuvers. We will also enroll 20 able-bodied age-matched control participants to capture age-specific norms for neurophysiologic measures.

Exclusion criteria across all participants are related to contraindications to TMS and tDCS, including cardiac pacemaker, metal in head and seizure history. In the case of participants with $\mathrm{SCl}$, presence of other conditions that can confound or adversely affect outcomes of rehabilitation is also exclusionary, including pressure injuries, associated traumatic brain injury, excessive tone/spasticity defined as Modified Ashworth Scale (MAS) >3, severe contractures, and ongoing UE therapies (see Table 1 for details).

Participants with $\mathrm{SCl}$ are randomly assigned to either $t D C S+$ rehab or shamtDCS + rehab group (see Fig. 1 for study design). In our pilot study, an effect size of 0.938 was achieved for the UE motor score (UEMS) between the two groups [8]. Based on this effect size, a sample of 40 is expected to provide $80 \%$ power at $a=0.05$ (with assumption of differences based on two sample $t$-statistics). We will recruit 44 participants (Cleveland Clinic: 17; Kessler Foundation: 14; Cleveland VAMC: 5, MetroHealth Rehabilitation: 8) to account for expected attrition.

\section{Participant recruitment and eligibility assessment}

Participants with tetraplegia are recruited using electronic medical record review, referrals, clinicaltrials.gov listing, and community outreach. The site coordinator completes preliminary eligibility screening over the phone. Candidates who meet preliminary eligibility undergo in-person eligibility assessment with site physician and site assessing therapist. The physician performs the examination as described by the International Standards for Neurological Classification of $\mathrm{SCl}$ to determine neurologic level and degree of incompleteness of injury (AIS) and confirms selection criteria [14]. The assessing therapist determines severity of motor impairment using UEMS which involves testing MRC of 5 key muscle groups in both extremities $[14,15]$. The assessing therapist confirms the presence of muscle imbalance between biceps and triceps. Able-bodied participants are only required to meet preliminary eligibility over the phone.

\section{Randomization and blinding}

Participants with $\mathrm{SCl}$ are randomized to $t D C S$ + rehab or shamtDCS + rehab groups using the covariate-adaptive randomization method [16]. This method balances the two groups on 3 baseline characteristics-MRC grade of weaker (triceps) muscle, AIS level (A, B, C, or D) and time postinjury ( $<5$ or $\geq 5$ years, cutoff based on prospect of favorable prognosis within first 5 years). Study biostatisticians (XW, ML) share a unique tDCS code for each participant with the site training therapist. The code is preset to deliver active or sham tDCS on a clinical-trial device with experimental blinding (see below). All investigators except the biostatistician and the training therapist are blinded to group assignment.

\section{Intervention}

Participants with $\mathrm{SCl}$ receive $t D C S$ + rehab or shamtDCS + rehab for fifteen, $2 \mathrm{~h}$ sessions, spread over 3 to 5 weeks. Our experience shows distributing sessions over several weeks improves adherence to and feasibility of participation, allowing patients to receive as few as three sessions per week to as many as five sessions per week based on preference and availability, and access to (para)transport resources. Our pilot study had indicated it was feasible for patients to come to the lab for therapies over ten study visits [8]. The motivation to increase number of visits to 15 was driven in part by evidence that more favorable effects of rehabilitation can be achieved with extended treatment. Participants in the previous study had also indicated an interest in receiving additional sessions in anticipation of greater, longer-lasting benefits, which also factored in into the decision of extending treatments.

UE rehabilitation is based on principles of massed-practice that involves performing reaching, pushing, pulling, lifting and grasping with multiple repetitions. Each task is performed at least 10 times with brief rest as 


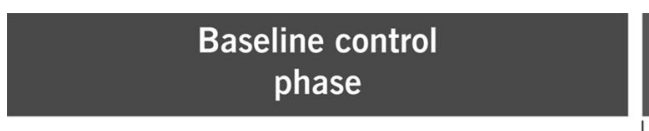
phase

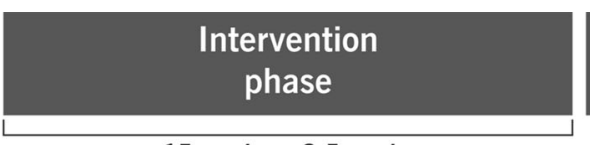

15 sessions, 3-5 weeks
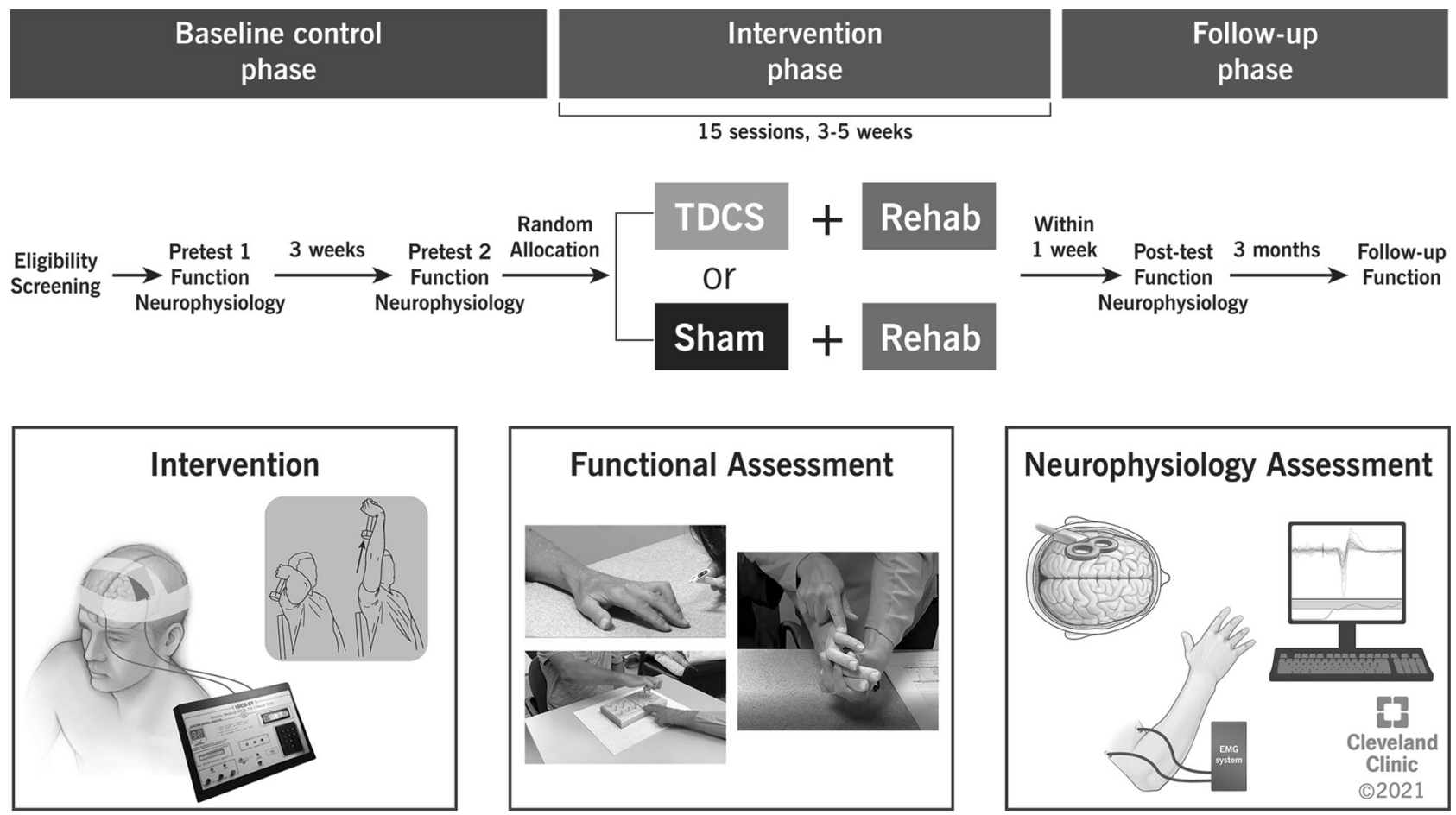

$\rightarrow$ Function $\longrightarrow$

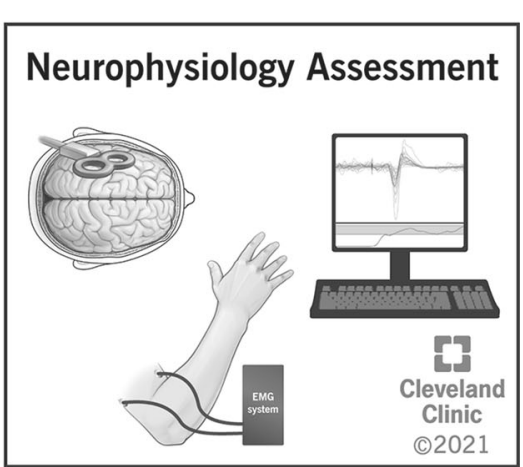

Fig. 1 Study design and follow-up procedures. TDCS transcranial direct current stimulation (tDCS).

needed. Eighty percent of tasks focus on the use of the more-affected UE, while $20 \%$ focus on the use of bilateral limbs. The training therapists at all sites have access to a Therapy Task Bank with various UE massed-practice movements. The training therapist selects tasks based on participant's level of impairment and individual goals. A 20 min rest break is provided between each hour of session. Participants are also asked to complete a 30 $\min \times 5$ days/week home exercise-program and an activity log designed to promote use of the more impaired UE in functional tasks.

$t D C S$ is delivered at a dose of $2 \mathrm{~mA}$ using a clinical-trial tDCS device designed to have blinding across both the investigator and the patient $(1 \times 1$ CT, Soterix Medical Inc., USA $)$ [17]. Silicon electrodes are placed in saline-soaked sponges $(5 \mathrm{~cm} \times 7 \mathrm{~cm}) \quad$ (EASYpad $^{\mathrm{TM}}$, Soterix Medical Inc.), with the anode centered over the TMS motor hotspot for weaker muscle and the return electrode placed over the contralateral supraorbital region (details below). Stereotactic neuro-navigation is used to guide placement of the anode and the location of the center and corners of electrode is marked daily to ensure consistency. The training therapist uses the unique tDCS code to deliver assigned anodal or sham stimulation. Stimulation is delivered for the first half-hour of each hour of the $2 \mathrm{~h}$ session, consistent with length of time for which safety of tDCS has been demonstrated in multi-session studies $[12,18]$. For sham, current ramps-up slowly to $2 \mathrm{~mA}$ over $30 \mathrm{~s}$, and then ramps-down slowly over the next $30 \mathrm{~s}$, at both the start and the end of stimulation, a method consistent with delivering placebo given its potential to elicit similar sensations on the scalp as active tDCS [19]. At the end of each session, participants are asked to guess whether they received active or sham stimulation. This information is used to assess blinding integrity.

\section{Functional outcomes assessments}

The site assessing therapist performs functional outcomes assessments, twice at baseline, once at end-of-intervention, and once at 3-month followup. Measures of motor impairment, functional abilities, and participation restrictions are collected in accordance with the International Classification of Functioning, Disability and Health, developed by the World Health Organization [20]. The assessing therapists at all sites are trained by a highly experienced occupational-therapy scientist (AMB). All assessing therapists meet regularly over conference calls to discuss any issues related to outcomes assessments.

Motor impairment is measured using Graded Redefined Assessment of Strength Sensibility and Prehension (GRASSP, version 1.0) designed specifically for UE assessment in tetraplegia. GRASSP includes standardized assessment of muscle power, sensation, and prehension capabilities. We will use the quantitative prehension capability as the primary outcome, whereas muscle power, sensation, and qualitative prehension scores will be used as secondary outcomes. GRASSP has excellent test-retest reliability (ICC $=0.86-0.98$ ), and adequate-to-excellent concurrent validity when used for the assessment of UE function in tetraplegia [21].

Functional abilities are measured using the Capabilities of upper extremity-test (CUE-T) [22], which consists of 19 tasks, 17 unilateral and 2 bilateral. Tasks involve a range of UE movements performed across proximal and distal joints for assessment of gross and fine motor function. Scoring for each item is based on ability to complete, rate of movement and time taken. CUE-T has excellent test-retest reliability $(I C C=0.97-0.98)$ and good-to-excellent concurrent validity (0.55-0.83) in SCI [22].

Patient-reported independence is measured using the Spinal Cord Independence Measure Ver. III (SCIM), which assesses the perceived ability to complete activities of daily living in 3 domains-self-care, respiration, and sphincter management, and mobility [23]. Items from the self-care domain (feeding, grooming, bathing and dressing) are tracked at longitudinal time points because of their relevance to UE motor function. SCIM has adequate-to-excellent interrater reliability $(\mathrm{ICC}=0.63-0.97)$ and excellent concurrent validity (0.85) [23].

Patient-perceived participation restrictions is measured using the Canadian Occupational Performance Measure (COPM), which tracks five most important occupational activities spanning across domains of self-care, productivity, and leisure. Patients rate their level of performance and satisfaction with each activity [24]. Test-retest values for performance and satisfaction show excellent reliability $(\mathrm{ICC}=0.99$ and 0.98 , respectively) [25].

\section{Transcranial magnetic stimulation (TMS)}

TMS is delivered using a figure-of-eight coil attached to a monophasic stimulator (diameter $70 \mathrm{~mm}$, Magstim 2002, Dyfed, UK). Surface electromyography (EMG) is collected from biceps and triceps on the moreaffected (test) side to record TMS-evoked motor potentials (MEPs) and other effects. If both sides have equal UEMS scores, patient-reported weaker side is tested. Motor cortical sites contralateral to the moreaffected UE are targeted with guidance from frameless stereotactic neuronavigation (Polaris Infrared tracking camera system, Northern Digital, Waterloo, ON, Canada).

We first identify a motor hotspot for each muscle. Motor hotspot is defined as a site that elicits criterion-sized MEPs (peak-to-peak $\geq 100 \mu \mathrm{V}$ above baseline EMG) in slightly contracted muscle (15-25\% of maximum voluntary contraction, MVC) at the lowest TMS intensity in $\geq 6 / 10$ 
consecutive trials [26]. The lowest TMS intensity used is called active motor threshold (AMT). AMT is a measure of corticomotor excitability [26]. Corticomotor gain and output are also measured using what is known as a Recruitment Curve (RC). RC involves recording MEPs at higher increments of intensities starting from $90 \%$ AMT (10\% increments, randomized) until no further increase in MEP size is seen [27]. Slope of an RC curve defines gain while area defines overall corticomotor output [28]. Corticomotor inhibition is also captured using cortical silent period (CSP) method, involving application of suprathreshold TMS (120\% AMT) to transiently interrupt ongoing EMG activity in slightly contracted muscle (15-25\% MVC) for 30 trials [29]. Corticomotor organization is evaluated using motor maps. Five suprathreshold TMS pulses each $(100 \%$ maximum stimulator output, MSO) are delivered to sites on a $7 \times 7$ grid $(10 \mathrm{~mm}$ resolution) centered at biceps motor hotspot in resting state of the muscle [30]. Number of sites that produce criterion MEPs (at least one-eighth of maximum MEP amplitude) and total amplitude of normalized MEPs (normalized to maximum MEP amplitude) are noted as area and volume, respectively [31].

\section{Safety and feasibility}

We assess safety based on type, severity and frequency of adverse event reports. Adverse events are categorized as-(1) tDCS-related (redness, dizziness, disorientation/confusion), (2) SCl-related (spasticity, clonus, pain, autonomic dysreflexia, or abnormal sensation), and (3) rehabilitationrelated (musculoskeletal injuries, abnormal changes in blood pressure, heart rate, or oxygen saturation levels). We also document TMS-related adverse events, though these are treated as separate from those related to the intervention ( $t D C S$ + rehab or sham + rehab). In addition, we also document unanticipated adverse events that may not be related to the intervention or study participation, such as community acquired COVID-19 infection, and falls, injuries, autonomic events or any other safety incidents not related to this research study. We document and report all adverse events to the IRB and the HRPO. Feasibility is defined in terms of attrition i.e., frequency of patients lost to follow-up. Feasibility also includes assessment of integrity of blinding. We note the frequency with which participants, or assessors are able to correctly guess group allocation.

\section{Data management}

Data is shared with the lead site using REDCap (all data except neurophysiology) and Lab Archives (neurophysiology). A data and safety monitoring board that consists of four external members (clinicians, scientists, lay reviewer) helps oversee safety and data integrity.

\section{Multisite management}

All sites are trained on the same training and testing modules for functional and neurophysiological assessments for standardization. Before the clinical-trial commenced, team members from all sites visited the lead site for training. Practical hands-on procedures were harmonized during the testing of the first two participants with $\mathrm{SCl}$ at each site. Monthly meetings are held across all sites to discuss study updates such as administrative issues, participant recruitment, data management, intervention, adverse events, or any challenges. The lead site reviews the quality of data collected at every site and provides feedback as necessary. The lead site has developed and continues to update standard operating procedures to ensure revised details of standardized procedures are available to all sites. Sites will be blinded to the data from other sites, except the lead site which will have access to data from all sites.

\section{Statistical plan}

For Aims 1 and 2, we will use a linear 2-way mixed-effects analysis of variance (GroupXTime) to evaluate changes in metrics of impairment, functional ability, participation restriction and TMS-based metrics. If the interaction is significant, we will compare the two groups at various time points using 2-sample $t$-test (with Bonferroni-correction). Since unilateral and bilateral scores can be obtained for GRASSP and CUE-T, we will perform a secondary analysis for aims 1 and 2 using unilateral scores for GRASSP and CUE-T. We will compare changes in absolute value of scores, which will allow us to make comparisons of improvements with the minimal clinically important differences for GRASSP, CUE-T, and COPM. To control for the variability within the participants, we will also conduct statistical comparisons on percentage improvements. For Aim 3, we will compare the continuous metrics, such as percent occurrence of adverse events between groups using a 2-sample $t$-test. For categorical metrics, such as frequency of attrition we will use Pearson's chi-square. Subanalyses will take into consideration different variables that can affect results such as level and completeness of injury, time since injury, age, handedness, lifestyle scores and testing site. Analysis plan will include intent-to-treat and per-protocol analysis approaches. Significance level will be set at $a=0.05$.

\section{REFERENCES}

1. National Spinal Cord Injury Statistical Center. Spinal cord injury facts and figures at a glance. Birmingham. AL: National Spinal Cord injury Statisical Center; 2016.

2. Simpson LA, Eng JJ, Hsieh JT. Wolfe and the Spinal Cord Injury Rehabilitation Evidence (SCIRE) Research Team DL. The health and life priorities of individuals with spinal cord injury: a systematic review. J Neurotrauma. 2012;29:1548-55.

3. Kloosterman MG, Snoek GJ, Jannink MJ. Systematic review of the effects of exercise therapy on the upper extremity of patients with spinal-cord injury. Spinal Cord. 2009;47:196-203.

4. Oudega $M$, Perez MA. Corticospinal reorganization after spinal cord injury. J Physiol. 2012;590:3647-63.

5. James ND, McMahon SB, Field-Fote EC, Bradbury EJ. Neuromodulation in the restoration of function after spinal cord injury. Lancet Neurol. 2018;17:905-17.

6. Nitsche MA, Cohen LG, Wassermann EM, Priori A, Lang N, Antal A, et al. Transcranial direct current stimulation: state of the art 2008. Brain Stimul. 2008;1:206-23.

7. Nitsche MA, Seeber A, Frommann K, Klein CC, Rochford C, Nitsche MS, et al. Modulating parameters of excitability during and after transcranial direct current stimulation of the human motor cortex. J Physiol. 2005;568:291-303.

8. Potter-Baker KA, Janini DP, Lin YL, Sankarasubramanian V, Cunningham DA, Varnerin NM, et al. Transcranial direct current stimulation (tDCS) paired with massed practice training to promote adaptive plasticity and motor recovery in chronic incomplete tetraplegia: a pilot study. J Spinal Cord Med. 2018;41:503-17.

9. Cortes M, Medeiros AH, Gandhi A, Lee P, Krebs HI, Thickbroom G, et al. Improved grasp function with transcranial direct current stimulation in chronic spinal cord injury. NeuroRehabilitation. 2017;41:51-9.

10. Yozbatiran N, Keser Z, Davis M, Stampas A, O'Malley M, Cooper-Hay C, et al. Transcranial direct current stimulation (tDCS) of the primary motor cortex and robot-assisted arm training in chronic incomplete cervical spinal cord injury: A proof of concept sham-randomized clinical study. NeuroRehabilitation. 2016;39:401-11.

11. Gomes-Osman J, Field-Fote EC. Cortical vs. afferent stimulation as an adjunct to functional task practice training: a randomized, comparative pilot study in people with cervical spinal cord injury. Clin Rehabil. 2015;29:771-82.

12. Murray LM, Edwards DJ, Ruffini G, Labar D, Stampas A, Pascual-Leone A, et al. Intensity dependent effects of transcranial direct current stimulation on corticospinal excitability in chronic spinal cord injury. Arch Phys Med Rehabil. 2015;96: S114-21.

13. De Araujo AV, Ribeiro FP, Massetti T, Potter-Baker KA, Cortes M, Plow EB, et al. Effectiveness of anodal transcranial direct current stimulation to improve muscle strength and motor functionality after incomplete spinal cord injury: a systematic review and meta-analysis. Spinal Cord. 2020;58:635-46.

14. Rupp R, Biering-Sørensen F, Burns SP, Graves DE, Guest J, Jones L, et al. International Standards for Neurological Classification of Spinal Cord Injury: revised 2019. Top Spinal Cord Inj Rehabil. 2021;27:1-22.

15. Marino RJ, Jones L, Kirshblum S, Tal J, Dasgupta A. Reliability and repeatability of the motor and sensory examination of the international standards for neurological classification of spinal cord injury. J Spinal Cord Med. 2008;31:166-70.

16. Winstein CJ, Miller JP, Blanton S, Taub E, Uswatte G, Morris D, et al. Methods for a multisite randomized trial to investigate the effect of constraint-induced movement therapy in improving upper extremity function among adults recovering from a cerebrovascular stroke. Neurorehabil Neural Repair. 2003;17:137-52.

17. Beaulieu LD, Blanchette AK, Mercier C, Bernard-Larocque V, Milot MH. Efficacy, safety, and tolerability of bilateral transcranial direct current stimulation combined to a resistance training program in chronic stroke survivors: a doubleblind, randomized, placebo-controlled pilot study. Restor Neurol Neurosci. 2019;37:333-46.

18. Potter-Baker K, Frederick F, Plow EB. It's all in your head: driving cortical plasticity to improve muscle contraction below the level of injury. J Spinal Cord Med. 2018;41:592.

19. Brunoni AR, Schestatsky $P$, Lotufo $P A$, Benseñor IM, Fregni F. Comparison of blinding effectiveness between sham tDCS and placebo sertraline in a 6-week major depression randomized clinical trial. Clin Neurophysiol. 2014;125:298-305.

20. World Health Organization. Towards a common language for functioning, disability, and health: ICF. The international classification of functioning, disability and health. 2002. Retrieved from https://www.who.int/classifications/icf/ icfbeginnersguide.pdf on Feb 28, 2022 
21. Kalsi-Ryan S, Curt A, Verrier MC, Fehlings MG. Development of the Graded Redefined Assessment of Strength, Sensibility and Prehension (GRASSP): reviewing measurement specific to the upper limb in tetraplegia. J Neurosurg Spine. 2012;17:65-76.

22. Marino RJ, Kern SB, Leiby B, Schmidt-Read M, Mulcahey MJ. Reliability and validity of the capabilities of upper extremity test (CUE-T) in subjects with chronic spinal cord injury. J Spinal Cord Med. 2015;38:498-504.

23. Itzkovich M, Gelernter I, Biering-Sorensen F, Weeks C, Laramee MT, Craven B, et al. The Spinal Cord Independence Measure (SCIM) version III: reliability and validity in a multi-center international study. Disabil Rehabil. 2007;29:1926-33.

24. Law M, Baptiste S, McColl M, Opzoomer A, Polatajko H, Pollock N. The Canadian occupational performance measure: an outcome measure for occupational therapy. Can J Occup Ther. 1990;57:82-7.

25. Berardi A, Galeoto G, Guarino D, Marquez MA, De Santis R, Valente D, et al. Construct validity, test-retest reliability, and the ability to detect change of the Canadian Occupational Performance Measure in a spinal cord injury population. Spinal Cord Ser Cases. 2019;5:1-8.

26. Rossini PM, Burke D, Chen R, Cohen LG, Daskalakis Z, Di lorio R, et al. Non-invasive electrical and magnetic stimulation of the brain, spinal cord, roots and peripheral nerves: basic principles and procedures for routine clinical and research application. An updated report from an IFCN Committee. Clin Neurophysiol. 2015;126:1071-107.

27. Carson RG, Nelson BD, Buick AR, Carroll TJ, Kennedy NC, Mac Cann R. Characterizing changes in the excitability of corticospinal projections to proximal muscles of the upper limb. Brain Stimul. 2013;6:760-8.

28. Devanne $\mathrm{H}$, Lavoie BA, Capaday C. Input-output properties and gain changes in the human corticospinal pathway. Exp Brain Res. 1997;114:329-38.

29. Chen R, Lozano AM, Ashby P. Mechanism of the silent period following transcranial magnetic stimulation evidence from epidural recordings. Exp Brain Res. 1999;128:539-42.

30. Brasil-Neto JP, McShane LM, Fuhr P, Hallett M, Cohen LG. Topographic mapping of the human motor cortex with magnetic stimulation: factors affecting accuracy and reproducibility. Electroencephalogr Clin Neurophysiol. 1992;85:9-16.

31. Potter-Baker KA, Janini DP, Frost FS, Chabra P, Varnerin N, Cunningham DA, et al. Reliability of TMS metrics in patients with chronic incomplete spinal cord injury. Spinal Cord. 2016;54:980-90.

\section{ACKNOWLEDGEMENTS}

The U.S. Army Medical Research Acquisition Activity, 820 Chandler Street, Fort Detrick MD 21702-5014 is the awarding and administering acquisition office. The authors would like to acknowledge the roles of all team members across study sites, including coordinators, therapists, research assistants, and engineers.

\section{AUTHOR CONTRIBUTIONS}

$E P B, K O, K P B, S K, K K, G F F, A B, X W, M A R$, SP, and FF were involved in generation of the idea and designing of the protocol. TA, EP, KO, KPB, SK, GFF, MKH, ML, KP, SP, and FB have been involved in protocol revisions and implementation. TA drafted the paper. All authors have been involved in revising and approving the final version of the paper.

\section{FUNDING}

Opinions, interpretations, conclusions and recommendations are those of the author and are not necessarily endorsed by the Department of Defense or U.S. Army. This work was supported by The Assistant Secretary of Defense for Health Affairs endorsed by the Department of Defense through the Spinal Cord Injury Research Program under Award No. W81XWH1810530. Here we report on version 1.0 of the protocol approved by the Cleveland Clinic Institutional Review Board (IRB), local site IRBs and the Department of Defense (DoD) Human Research Protection Office (HRPO).

\section{COMPETING INTERESTS}

The authors declare no competing interests.

\section{ETHICS APPROVAL AND CONSENT TO PARTICIPATE}

This study has received ethical clearance from IRB of each participating site and the HRPO of the U.S Army Medical Research and Development Command. All participants provide written informed consent before participating. The knowledge from this trial will be disseminated through conference presentations and publications. We aim to target $\mathrm{SCl}$ and rehabilitation forums to impact practice, education, research, and policy development.

\section{ADDITIONAL INFORMATION}

Correspondence and requests for materials should be addressed to Ela B. Plow.

Reprints and permission information is available at http://www.nature.com/ reprints

Publisher's note Springer Nature remains neutral with regard to jurisdictional claims in published maps and institutional affiliations. 